\title{
Turboprop Engine Fault Diagnosis Based on Hilbert Spectrum and Singular Value Decomposition
}

\author{
Feng Ding ${ }^{1, a^{*}}$ and Zhi Qi ${ }^{1, b}$ \\ ${ }^{1}$ Department of Mechanical and Electronic Engineering, Xi'an Technological University, Xi'an, China \\ adfeng@xatu.edu.cn, b13462208735@163.com \\ *The corresponding author
}

Keywords: Fault diagnosis; Feature extraction; Hilbert spectrum; Singular value decomposition; RBF network

\begin{abstract}
In order to solve the time-frequency feature extraction problem of the vibration signal, a fault diagnosis method based on Hilbert spectrum and singular value decomposition is proposed and applied to engine fault diagnosis. Firstly, the vibration signals are decomposed into a series of intrinsic mode functions by using empirical mode decomposition method. Secondly, Hilbert transform is applied to each intrinsic mode function and Hilbert spectrum of the vibration signal is got. Then the singular value method is applied to the Hilbert spectrum and the singular value vector is acquired. Finally, as feature vectors, singular value vectors are input into RBF network for identifying the different fault. Experimental simulation shows that this method can extract effectively the engine fault vibration signal characteristics.
\end{abstract}

\section{Introduction}

Aero-engine is the heart of the aircraft, so its reliable work is an important guarantee for airplane flight [1]. Therefore, it is very necessary for engine fault diagnosis to detect timely and accurately the potential and existential faults occurring in the engine and to guarantee the safe operation of the engine [2].

Fault diagnosis is essentially a problem of pattern recognition, and the feature extraction and selection is the key to pattern recognition [3]. Wavelet method can extract local information of time domain and frequency domain of the vibration signal at the same time, so it has been widely used in the field of fault diagnosis [4,5]. But the selection of wavelet basis function for wavelet analysis is a difficult problem. Literature [6] proposes an adaptive time-frequency analysis method which mainly uses empirical mode decomposition to get the intrinsic mode function. The method has achieved good results in the field of fault diagnosis. Literature [7] gets the intrinsic mode function of the gearbox vibration signal based on empirical mode decomposition, and then energy entropy of the intrinsic mode function is regarded as the fault feature. Literature [8] put forward a fault diagnosis method based on the singular value decomposition (SVD) of intrinsic mode functions and support vector machine (SVM), and this method decomposes vector formed by the intrinsic mode function based on the singular value decomposition method, and decomposition result is regarded as fault characteristic vectors.

SVD is a kind of important matrix decomposition, and the number of singular values reflects the number of independent row (column) vector in the original matrix. RBF network has the strong ability of mode identification and the data approximation, and has the information processing characteristics of self-study, self-adaptability, parallelism, robustness and redundancy, and widely used in fault diagnosis [9].

In this paper, the vibration signals are decomposed into several intrinsic mode functions components based on empirical mode decomposition method. Then, Hilbert transform is applied to each intrinsic mode function component and the instantaneous frequency and instantaneous amplitude of vibration signal are acquired, and then the Hilbert spectrum of vibration signal is obtained. The Hilbert spectrum is decomposed based on singular value decomposition method, and the result after decommission is used as the characteristic vector of engine fault diagnosis to input 
into the RBF neural network for pattern recognition, verifying the validity of the above feature extraction method.

\section{Fault Diagnosis Model}

The Hilbert Spectrum. Literature [7] proposes the EMD method that signals is decomposed into a series of IMF components. This process not only eliminates modal superposition of waveform, but also makes waveform contour more symmetrical. After the EMD decomposition, the original signal can be represented as:

$$
f(0) \sum_{i=1}^{n} i_{i} m f+h_{h}()
$$

Where $\operatorname{imf}_{i}(t)$ indicates intrinsic mode functions after the decomposition, $r_{n}(t)$ is the residual function. The Hilbert transform is applied to each intrinsic mode function and get:

$$
i \hat{m} f_{i}=\frac{1}{\pi} \int_{-\infty}^{\infty} \frac{i m f_{i}(\tau)}{t-\tau} d \tau
$$

Structure analytic signal:

$$
z_{i}(t)=\operatorname{imf}_{i}(t)+j i \hat{m} f_{i}(t)=a_{i}(t) e^{j \phi_{i}(t)}
$$

So we can get:

$$
x(t)=s r \sum_{i=1}^{n} a_{i}(t) e^{j \phi_{i}(t)}
$$

Where $s r$ indicates real component. Eq. 4 is called the Hilbert spectrum and marked as $H(\omega, t)$. $H(\omega, t)$ describes accurately changing rule of the amplitude of the signal with the time and frequency on the whole frequency.

The Singular Value Decomposition. The matrix of time-frequency distribution is marked as A which are obtained by the Hilbert spectrum of vibration signals, and $\mathrm{A}$ is assumed as a $m \times n$ order matrix. This paper, the singular values of matrix A, the time-frequency structure characteristic of the Hilbert spectrum matrix of signal, can be obtained based on the singular value decomposition to matrix A. The larger singular values contain more information about the structure of the vibration signal among them. Therefore, we can use the singular values of the Hilbert spectrum as the characteristics of the mechanical fault diagnosis.

Model Establishment. Combing EMD decomposition, Hilbert transform, SVD method and RBF neural network to establish engine fault diagnosis model which is shown in Fig. 1. The vibration signals are decomposed into several IMF components based on EMD method; Hilbert spectrum is obtained based on Hilbert transform applied to IMF components; the Hilbert spectrum is decomposed by SVD method; the singular values are input into RBF neural network for training and testing. 


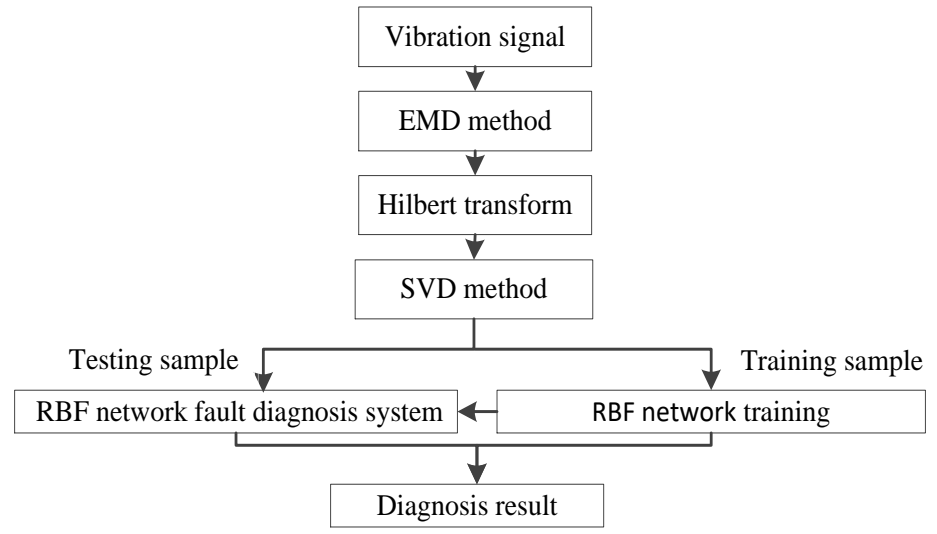

Figure 1. Engine fault diagnosis model

\section{Application}

The application data comes from the China national aviation company in this paper. Vibration experiment is conducted on instance engine test bed in the factory, and experiment uses DT9837 type data acquisition instrument [10]. Engine speed is $12300 \mathrm{r} / \mathrm{min}$, and the sampling frequency is $2 \mathrm{KHz}$. The data set includes 3 subsets that are the new engine subset, the long test engine (it has run for 600h) subset, the fault engine subset, and each subset corresponds to a state, containing 20 samples. Among the 120 samples, 90 samples are for training, and the remaining 30 samples are for testing, and the sampling points of each sample is 8192. The time domain waveform of vibration signals of engine three states are shown in Fig. 2. Engines' three states are shown in Table 1.

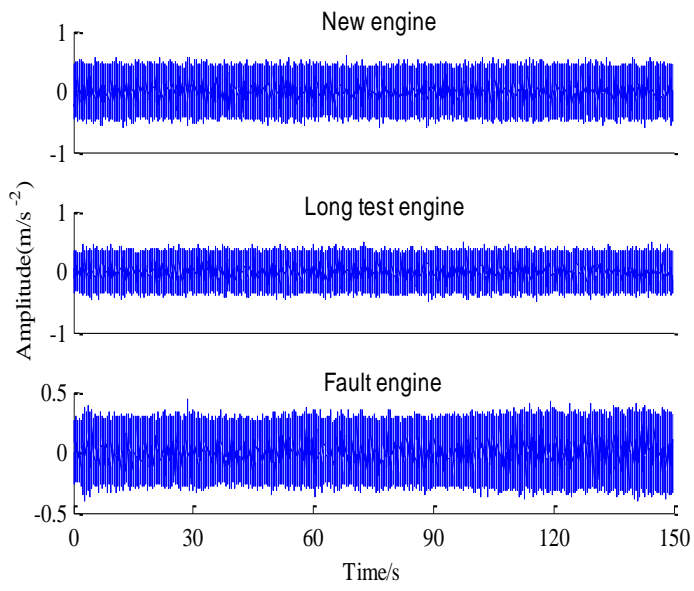

Figure 2. The vibration signal's time domain waveform of three kinds of engine state

Table 13 states of engine

\begin{tabular}{|c|c|c|c|}
\hline The number of training sample & The number of testing sample & engine states & Label \\
\hline 30 & 10 & new engine & 1 \\
\hline 30 & 10 & long test engine & 2 \\
\hline 30 & 10 & fault engine & 3 \\
\hline
\end{tabular}

In the test, the vibration signals are decomposed by EMD method, and IMF components are obtained. Hilbert transform is applied to the former ten IMF components extracted and the Hilbert spectrum is obtained. The Hilbert spectrum is decomposed by SVD method, and the result after 
decommission works as the characteristics information of the engine fault diagnosis. The former 50 singular value curve of vibration signal under the new state, long commissioning state and fault state of engine is shown in Fig. 3.

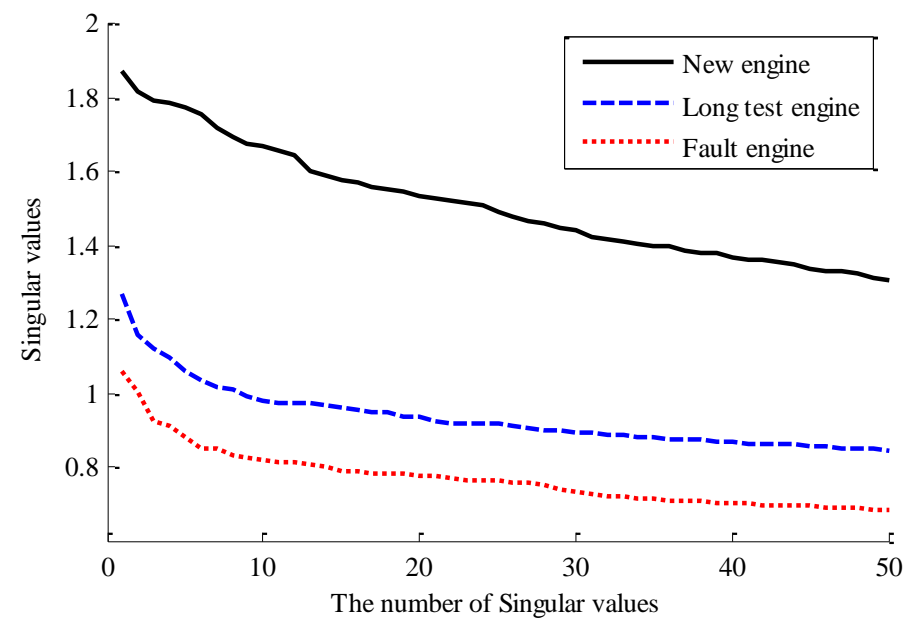

Figure 3. The singular value curve of Hilbert spectrum of vibration signal under 3 three states

As seen from Fig. 3, singular values of the Hilbert spectrum of vibration signals are significantly different when the engine is under different states. Singular values are largest under new engine condition, and singular values become smaller after running for $600 \mathrm{~h}$, and singular values are minimum when the engine is failure. So the singular values of the Hilbert spectrum of the engine vibration signal can be used as the characteristics of the engine fault diagnosis.

The former 20 singular values of the Hilbert spectrum are used as fault feature to input into RBF neural network for training and testing. The number of nodes in input layer and output layer of RBF network are 20 and 1 , setting the spread of RBF neural network is 0.2 . The classification accuracy of RBF neural network is shown in Table 2.

Table 2 The classification accuracy of RBF neural network

\begin{tabular}{cccccccc}
\hline \multirow{2}{*}{ Network types } & \multicolumn{2}{c}{ Training accuracy rate $(\%)$} & \multicolumn{3}{c}{ Testing t accuracy rate $(\%)$} \\
\cline { 2 - 7 } & Min & Mean & Max & Min & Mean & Max \\
\hline \multirow{2}{*}{ RBF neural network } & 86.63 & 97.98 & 100 & 87.84 & 98.07 & 100 \\
\hline
\end{tabular}

As shown in Table 2, after the pattern recognition based on RBF neural network, the average training accuracy rate and testing accuracy rate is $97.98 \%$ and $98.07 \%$, respectively; the minimum training and test accuracy rate is $86.63 \% \%$ and $87.84 \%$, respectively. A higher recognition rate achieved for fault diagnosis illustrates that the method based on singular value of Hilbert spectrum for turboprop engine fault diagnosis is effectiveness.

\section{Conclusion}

The Hilbert spectrum contains two aspects of information in time domain and frequency domain. Combining with the singular value decomposition method, time-frequency characteristics extraction of the Hilbert spectrum is realized. This paper, fault feature information of turboprop engine extracted based on the singular value of Hilbert spectrum provides sensitive and effective characteristic parameters for engine state recognition. Through the pattern recognition of the measured signal of turboprop engines under the new state, long text state and fault state, it is verified that the proposed method in this paper can extract accurately the engine fault feature. 


\section{Acknowledgements}

This research is financially supported by the National Science Foundation of China (No. 51275374) and the Fund Project of Science \& Technology on Reliability \& Environmental Engineering Key Laboratory.

\section{References}

[1] H.W. Wang, J. Gao and H.Q. Wu: Reliability Analysis on Aero-engine Using Bayesian Model Averagin, Journal of Aerospace Power, (2014) No.02, p.305, (In Chinese).

[2] Z.W. Wangi, Y. Hu and Y.W. Li: Fault Diagnosis of the Rotating Equipment Based on Wavelet Analysis and Adaptive Fuzzy Neural Network, Journal of East China Jiaotong University, (2010) No.01, p.72, (In Chinese).

[3] H. Guo, L.B. Jack and A.K. Nandi: Feature Generation Using Genetic Programming with Application to Fault Classification, IEEE Transactions on Systems, Man, and Cybernetics, Part B: Cybernetics, (2005), p.351.

[4] Cu, L.X. Gao and ect: Research on Composite Fault Diagnosis Method Based on the Second Generation Wavelet, Chinese Journal of Mechanical Engineering, (2009) No.04, p.442, (In Chinese).

[5] F.B. Cheng, B.P. Tang and L. Zhao: A Filtering Method Based on Optimal Morlet Wavelet and Application in Machine Fault Feature Analysis, Chinese Journal of Mechanical Engineering, (2009) No.12, p.1437, (In Chinese).

[6] N.E. Huang, Z. Shen, S.R. Long and et al: The Empirical Mode Decomposition and the Hilbert Spectrum for Nonlinear and Non-stationary Time Series Analysis (London, 1998). P. 903.

[7] C. Zhang, J.J. Chen and X. Guo: A Gear Fault Diagnosis Method Based on EMD Entropy and SVM, Journal of Vibration and Shock, (2010) No.10, p.216, (In Chinese).

[8] Q. Gao, X.S. Du and et al: An Empirical Mode Decomposition Based Method for Rolling Bearing Fault Diagnosis, Journal of Vibration Engineering, (2007), N0.01, p.15, (In Chinese)

[9] H. Simon: Neural Networks a Comprehensive Foundation (China Machine Press, China 2003).

[10]L. Cheng, K. Qu, W. Cheng and et al: Vibration Monitoring Threshold of Turboprop Engine Reducer, Journal of Vibration and Shock, (2015) No.18, p.136, (In Chinese). 